\title{
SOGGETTIVITÀ, UMANO E TECNICHE DEL SÈ: L'ULTIMO FOUCAULT E IL SECONDO HEIDEGGER
}

\author{
SUBJECTIVITY, HUMAN AND TECHNIQUES OF SELF: THE LAST FOUCAULT AND THE SECOND \\ HEIDEGGER
}

Graziano Mazzocchini*

\section{RESUMO}

Em duas contribuições do "último" Foucault, a saber, o Curso ministrado ao Collège de France em 1982, A hermenêutica do sujeito, e a última entrevista por ele concedida, Le retour de la morale, o filosofo francês atesta expressamente o papel fundamental desempenhado pela influência do pensamento de Heidegger ao longo da sua inteira elaboração filosófica. Os dois textos mencionados acima ressaltam, justamente, na ontologia heideggeriana uma das matrizes a partir das quais Foucault refletiu sobre a recíproca relação entre sujeito e verdade, por sua vez constitutiva da questão da subjetividade. Além desse grupo de textos há ainda outro texto-chave, constituído pela Introdução ao segundo volume da foucaultiana História da sexualidade, O uso dos prazeres (1984): ao reconsiderar todo o seu itinerário filosófico, Foucault reinterpreta as suas investigações acerca dos "jogos de verdade", isto é, das maneiras contingentes com as quais uma determinada subjetividade se forma e se capta em relação a determinadas práticas de veridiction, como uma série de problematizações através das quais "o ser se dá como podendo e devendo ser pensado", retomando assim literalmente, na sua "história crítica do pensamento", a instância fundada pelo Heidegger da "virada" de indagar a "historicidade do ser" nas maneiras com as quais este último se doa à interpretação do ente humano. Em conclusão, teremos fundamentado a tese segundo a qual é licito vislumbrar uma semelhança entre a "história do ser" esboçada por Heidegger depois de Ser e tempo e a história foucaultiana dos jogos de verdade, mas ao mesmo tempo veremos como a tematização foucaultiana das "técnicas do si mesmo" acabe derrubando o sentido da estrutura ontológica heideggeriana.

PALAVRAS-CHAVE: Foucault. Heidegger. História do ser. História crítica do pensamento. Técnicas.

\footnotetext{
* Doutorando Programa de Pós-graduação em Filosofia da UFMG. E-mail: grazianomazzocchini@gmail.com.
} 


\section{ABSTRACT}

In two contributions of the "last" Foucault, namely the Course taught at the Collège de France in 1982, The Hermeneutics of the subject, and the last interview granted by him, Le retour de la morale, the French philosopher expressly emphasized the role played by the influence of Heidegger's thought throughout his entire philosophical elaboration. Against the background of Heidegger's ontology, which constitutes the very source of Foucault's theoretical investigation, the French philosopher reflects on the reciprocal relationship between subject and truth, for its turn constitutive of the question of subjectivity. In addition to this group of texts there is another key text, consisting of the introduction to the second volume of Foucault's History of Sexuality, The use of pleasure (1984): to reconsider all his philosophical itinerary, Foucault reinterprets his research about "games of truth ", i.e., the contingent ways in which a given shape subjectivity forms itself and is captured for certain practices of veridiction, as a series of problematizations through which "being is given as that which can and must be thought". This kind of milestone allows Foucault to get back to his critical history of thought, so as to regain the instance of the Heidegger's "turn", consisting in querying the historicity of being, according to the ways with which the same being offers itself to the interpretation operated by the human "entity". In conclusion we support the thesis which settles down the possibility to envisage a similarity between the "history of being" sketched by Heidegger after Being and time and the Foucault's history of the games of truth. At the same time, however, this similarity offers the chance to notice how Foucault's thematization of the "techniques of himself" finally overturns the structure of the Heidegger's ontology.

KEY-WORDS: Foucault. Heidegger. History of being. Critical history of thought. Techniques.

Nel mettere a fuoco le questioni tra loro interrelate della formazione della soggettività e delle tecniche od arti della costituzione del sè nell'ultimo Foucault, ossia nel tratto della sua opera (1978-1984) incentrato da un lato sull'istanza di un'etica per una formazione del soggetto concepita in termini precipuamente estetici ${ }^{1}$, e dall'altro- e correlatamente- su di una

\footnotetext{
${ }^{1}$ Foucault ci attesta nel secondo (1984) dei due scritti dedicati alla questione, inaugurata da Kant nel 1784, circa cosa sia l'Illuminismo, che il criterio in base al quale il soggetto della modernità si coglie nella sua stessa costituzione presente è appunto analogo a quello, descrittoci da Charles Baudelaire nel secolo XIX, proprio dell'artista Constantin Guys, il quale coglieva i soggetti dei suoi quadri in procinto di divenire qualcosa d'altro
} 
riconsiderazione strategica del pensiero genealogico in vista di una costituzione "in libertà" del soggetto stesso, non ci si può non imbattere in un nodo filologico prezioso per la stessa decifrazione della filosofia di Foucault: quella dell'influenza, ora scoperta ora sotterranea, dell'ontologia di Heidegger sull'ontologia critica foucaultiana "di ciò che siamo" o del "nostro essere attuale" (FOUCAULT, 1998). Chiaramente presente come pietra di paragone diretta nei primi scritti degli anni Sessanta- e in particolar modo ne Le parole e le cose (1966) e nella tesi dottorale complementare sull'Antropologia pragmatica di Kant ${ }^{2}$,durante quest'ultima fase "etica" incentrata sulla costituzione della soggettività tale interlocuzione si presenta probabilmente come più rarefatta, e tuttavia niente affatto indimostrabile; a suffragare l'ipotesi di una costante, ancorchè nascosta, influenza del pensatore tedesco su quello francese stanno due notizie risaltenti proprio a quel periodo, delle quali prima per ordine di importanza è l'ultima intervista rilasciata da Foucault nel 1984, Il ritorno della morale (Le retour de la morale):

[...] Heidegger è sempre stato, per me, il filosofo fondamentale. Ho cominciato leggendo Hegel, poi Marx, e mi sono messo a leggere Heidegger nel '51 o nel '52; e nel ' 53 , o nel ' 52 , non mi ricordo più, ho letto Nietzsche. Conservo ancora gli appunti che avevo preso mentre leggevo Heidegger- ne ho delle montagne! - e sono molto più importanti di quelli che avevo preso su Hegel o su Marx. Tutto il mio divenire filosofico è stato determinato dalla lettura di Heidegger. [...] Non conosco a sufficienza Heidegger, praticamente non conosco nè Essere e tempo, nè le cose che sono state pubblicate recentemente. La mia conoscenza di Nietzsche è sicuramente migliore di quella che ho di Heidegger; nondimeno, sono le due esperienze più fondamentali che ho fatto. Probabilmente, se non avessi letto Heidegger, non avrei letto Nietzsche. Avevo provato a leggere Nietzsche negli anni Cinquanta, ma Nietzsche da solo non mi diceva nulla. Mentre Nietzsche e Heidegger sono stati uno schock filosofico! Ma non ho mai scritto nulla su Heidegger e su Nietzsche ho scritto solo un breve articolo; tuttavia sono i due autori che ho letto di più. Credo che sia importante avere alcuni autori con cui si pensa, con cui si lavora, ma su cui non si scrive. (FOUCAULT, 1998, p. 268-269; corsivi miei).

\footnotetext{
da come essi erano. Ne consegue che per l' "ontologia del nostro essere attuale" o per la "critica di noi stessi", abbozzata da Foucault in questo come nell'altro scritto, il processo di soggettivazione muova da una volontà a sottrarsi a quei moduli di veridizione nei quali il soggetto si trova fissato, e che nel farlo esso si colga secondo altre possibilitá che gli si prospettano esattamente nel momento in cui riflette sul proprio momento presente secondo un'immagine di ció in cui desidererebbe divenire per costituirsi nel maggior grado possibile di libertà. Secondo tale tratteggio, l'ontologia storica di noi stessi capovolge la prosepttiva kantiana di una limitazione necessaria a priori della conoscibilitá nostra e del mondo in un insieme di prove, definite da Foucault "storicocritiche", attraverso le quali le soggettivitá sperimentano i propri limiti al fine di una loro possibile forzatura verso ulteriori possibilitá del sè. Entrambi gli scritti sono antologizzati in FOUCAULT 1998.

${ }^{2}$ Sebbene nell'Introduzione all' Antropologia di Kant (FOUCAULT in KANT 2010) non vi sia nessun riferimento esplicito a Heidegger, si può tuttavia agevolmente rintracciare un richiamo all'analitica esistenziale heideggeriana nel riferimento alla ripetizione delle possibilità originarie dell'Esserci nel concreto rapportarsi di quest'ultimo al "mondo".
} 
La seconda conferma la ricaviamo dal dibattito che fa da intervallo alla lezione del 3 febbraio del Corso del 1982 sull' Ermeneutica del soggetto, durante il quale Foucault menziona l'opera heideggeriana come il precedente principale della sua stessa problematizzazione o storia dei rapporti tra soggettività e verità, o meglio come vera e propria falsariga della riflessione che concerne le "tecniche" o "tecnologie del sè"(FOUCAULT 2004), che il soggetto esercita su se stesso così da prodursi storicamente quale soggettività determinabile:

\begin{abstract}
In questo caso, potremmo affermare che non sono stati molti quelli che, nel corso degli ultimi anni - direi: nel corso del xx secolo - hanno sollevato la questione della verità. Non sono stati molti i pensatori che hanno posto domande come queste: che ne è del soggetto e della verità? E ancora: che ne è del rapporto del soggetto con la verità? Che cosa è il soggetto della verità, che cosa è il soggetto che dice il vero, e così via? Personalmente, riesco a individuarne solo due: Heidegger e Lacan. E per quanto mi riguarda - forse lo ha sentito dire - è piuttosto sul versante di Heidegger, e a partire da lui, che ho tentato di riflettere su tutto ciò. (FOUCAULT 2004, p.166).
\end{abstract}

\title{
II
}

Posta già sicuramente, in termini filologici, la decisiva influenza dell'ontologia heideggeriana sull'intera parabola filosofica foucaultiana procediamo dunque a specificare come si articoli la più esplicita ripresa, da parte del filosofo francese, degli interrogativi posti all'ontologia e all'umanismo filosofico dal nuovo corso intrapreso dal filosofo tedesco in seguito alla cosiddetta "svolta" (Kehre), successiva a Sein und Zeit, a partire dalla Lettera sull'umanismo (1946) di Heidegger a Jean Beaufret e lungo gli scritti pubblicati per la prima volta nel 1954, segnatamente in La questione della tecnica. Sia quest'ultimo saggio che la Lettera risultano certamente tra le letture di Heidegger condotte da Foucault a partire degli anni Cinquanta: del primo segnatamente sappiamo che costituì un testo di studio e riflessione costanti per Foucault, al pari della Crisi delle scienze europee di Husserl ${ }^{3}$-, nel mentre il secondo scritto in questione fu recepito dal filosofo francese attraverso le stesse lezioni di Beaufret, destinatario della Lettera e tra coloro che introdussero il pensiero heideggeriano in Francia - lezioni che Foucault seguì assiduamente e con interesse a partire dal suo ingresso

\footnotetext{
${ }^{3}$ Così ci riferisce in una nota a L'Ermeneutica del soggetto il suo curatore, Frèdèric Gros (FOUCAULT, 2004, p. 557n.).; in particolare, la Crisi delle scienze europee di Husserl presenta per la prima volta l'espressione " $a$ priori storico", che Foucault mutuerà ma al contempo modificandolo profondamente, giacchè per Husserl esso designava ancora una strutturale unità di senso dell'intero corso storico, mentre per il filosofo francese esso vale come principio di strutturazione spaziale nella coordinazione sguardo-oggetto clinico in Nascita della clinica (1964), e poi come principio immanente ai discorsi nel loro strutturarsi e del loro rendersi intelligibili quali discorsi veridici of falsificabili ne L'Archeologia del sapere (1969).
} 
nel 1946 all'Ècole Normale Supèrieure in Rue d'Ulm a Parigi - notizia, quest'ultima, riportataci da Didier Eribon nella sua biografia intellettuale di Foucault (ERIBON, 1990). Prendiamo dunque le mosse esattamente dalla Lettera per due ragioni tra loro correlate: perchè a questa precisa altezza della riflessione heideggeriana la costitutiva "apertura" dell'Esserci quale essere-nel-mondo si con-verte, subisce una torsione nel senso di un ribaltamento prospettico del riferirsi dell'Esserci alle strutture ontologiche fondamentaliquali erano state delucidate in Sein und Zeit - nel riferimento dell'essere all'Esserci (“dell'essere all'essere umano") - nel senso, cioè, del costitutivo "stare dentro" dell'Esserci nell' essere, sicchè Heidegger può qui affermare che il primo dimora, "abita" nel secondo- , e perchè la riflessione sulla "tecnica" come "destino dell'essere" in cui si dis-vela la stessa "verità" dell'essere nella sua dimensione strutturalmente "storica", quale si manifesta nell' "homo humanus" (HEIDEGGER, 2013), è qui introdotta e viene a formare esattamente la "traccia di riflessione" direttamente ripresa da Foucault a proposito dello stesso tema delle tecniche producenti il sè della soggettività. "Verità" e " tecniche": questi sono i due lemmi che pertanto dobbiamo qui tenere presenti; si tratta infatti di due termini-chiave tanto dell'ontologia heideggeriana quanto dell'etica estetica di Foucault, e nei quali lo stesso Foucault è disposto a riconoscere la specifica ereditá heideggeriana entro il suo progetto di una "storia critica del pensiero". Quest'ultima definizione della propria opera è data, in una sorta di retrospettiva che vuole abbracciarne l'intera parabola, dallo stesso Foucault nella voce dedicata al suo pensiero nel Dictionnaire des philosophes, curato da Denis Huisman e edito da PUF nel 1984, e sta ad indicare

un' analisi delle condizioni in cui si formano o vengono modificate certe relazioni tra il soggetto e l'oggetto, nella misura in cui queste ultime sono costitutive di un sapere possibile. Non si tratta di definire le condizioni formali del rapporto con un oggetto; non si tratta neanche di cogliere le condizioni empiriche che, a un determinato momento, hanno potuto permettere al soggetto in generale di prendere conoscenza di un oggetto già dato nel reale. La questione consiste nel determinare ciò che deve essere il soggetto, a quale condizione è sottomesso, quale statuto deve occupare nel reale o nell'immaginario, per diventare soggetto legittimo di questo o di quel tipo di conoscenza ; si tratta, insomma, di determinare il suo modo di "soggettivazione"...Ma la questione consiste anche, e nello stesso tempo, nel determinare a quali condizioni qualcosa può diventare un oggetto di conoscenza possibile, come ha potuto essere problematizzato in quanto oggetto da conoscere, a quale procedura di delimitazione ha potuto essere sottoposto...Si tratta, dunque, di determinare il suo modo di oggettivazione, che non è, neanche esso, lo stesso a seconda del tipo di sapere di cui si tratta. Questa oggettivazione e questa soggettivazione non sono indipendenti l'una dall'altra; è dal loro vicendevole sviluppo e dal loro legame reciproco che nascono quelli che potrebbero essere definiti "giochi di verità": cioè, non la scoperta delle cose vere, ma le regole in base a cui quello che un soggetto può dire a proposito di certe cose rientra nella questione 
del vero e del falso. La storia critica del pensiero ...è la storia dell'emergere dei giochi di verità; è la storia delle "veridizioni", intese come le forme, secondo cui, in un ambito di cose, si articolano i discorsi suscettibili di essere definiti veri o falsi...[...] Attualmente Michel Foucault ha iniziato...sempre all'interno dello stesso progetto generale, a studiare la costituzione del soggetto come oggetto per se stesso: la formazione delle procedure con cui il soggetto è portato a osservare se stesso, a analizzarsi, a decifrarsi, a riconoscersi come ambito di sapere possibile. Si tratta, insomma, della storia della "soggettività", intendendo con questo termine il modo in cui il soggetto fa esperienza di se stesso in un gioco di verità in cui è in rapporto con sè. (FOUCAULT, 1998, p. 248-249 e 250; corsivi miei).

Da tale illustrazione di cosa significhi, per Foucault, "storia critica del pensiero", e da quanto già accennato circa il mutamento di prospettiva allora occorso nella problematica ontologica del filosofo tedesco, riusciamo già ad intravedere la suddetta "traccia heideggeriana" offerta al filosofo francese dalla Lettera sull'umanismo: tale storia critica del pensiero, in quanto storia dei singolari modi di apparizione contestualmente della soggettività e della conoscenza “oggettiva”, presenta un'analogia con la "storia dell'essere" delineata nello scritto heideggeriano in questione, nel quale è appunto l'essere che ad-viene all'Esserci in virtù dello statuto ontologico costitutivamente "aperto" di quest'ultimo, presentandosi così necessariamente e strutturalmente quale storia del suo stesso avvenire. Di più, tale avvenire dell'essere all'ente, o tale “con-venire” dell'essere con l'essente (l'ente, appunto) mediante il "linguaggio" in cui letteralmente si destina, non è qui pensato da Heidegger alla stregua di una realizzazione di un'idea, di un'essenza orginaria ed originariamente depositata nella realtà ontica, bensì appunto quale "avvento" (Ankunft) od "evento" (Ereignis), il che definisce la stessa presentazione della verità all'essere-nel-mondo in termini analoghi a quell'evenemenzializzazione $e^{4}$ in virtù della quale, per Foucault, emergono od appaiono i giochi di verità, $\mathrm{o}$ - in una prospettiva ancora più marcatamente "genealogica" - i giochi di saperi e poteri, a partire dai quali soltanto possono interpretarsi tanto il soggetto quanto gli oggetti (FOUCAULT, 1997): lì il pervenire o manifestarsi della verità dell'essere, qui l'emergere dei giochi di verità si offrono parimenti alla stregua di eventi. Così ad esempio Heidegger, nella Lettera a Beaufret, delinea il movimento della verità dell'essere verso il

\footnotetext{
${ }^{4}$ Così Foucault definisce tale procedura nella conferenza su Illuminismo e critica (1978):" Per procedura di evenemenzializzazione...intenderei questo: innanziatutto prendere in considerazione insiemi di elementi in cui individuare, in via del tutto empirica e provvisoria, delle connessioni tra meccanismi di coercizione e contenuti di conoscenza. Meccanismi di coercizione diversi, quali insiemi legislativi, regolamenti, dispositivi materiali, espressioni di autorità etc.; contenuti di conoscenza diversi, eterogenei che occorrerá considerare in funzione degli effetti di potere di cui sono portatori e per il fatto di essere convalidati dall'appartenenza a un sistema di conoscenza"(FOUCAULT 1997, p. 53). Si tratta, in altre parole, di una descrizione storica a-valutativa attraverso la quale far apparire o risaltare connessioni affatto contingenti tra determinati saperi o moduli di veridizione, da un lato, e determinate relazioni di potere, dall'altro, nella cui intersezione si definisce e puó modificarsi una determinata soggettivitá, tanto nel suo rapporto con se stessa quanto nel suo rapporto con gli oggetti.
} 
pensiero proprio nei termini di un adveniens o ancora di un darsi, di un concedersi; sicchè, posto che .l'essenza dell' esistenza umana coincide qui con la verità dell'essere, l'esistenza stessa dell'uomo viene a configurarsi non già- in termini metafisici- quale entelechia o attualizzazione di una virtualità già riposta in se stessa, bensì, in quanto riceve la sua verità dall'essere, quale "estatico stare dentro nella verità dell'essere", quale "e-sistenza" (HEIDEGGER, 2013). Di qui l'eclissarsi dell'humanitas intesa nel senso dell'umanismo filosofico in tutte le sue varianti (rinascimentale,hegeliana, marxiana): la verità od essenza dell' homo humanus non riposa, per Heidegger, su di un'essentia situata dentro l'ente-uomo di cui la sua existentia costituisca un'estrinsecazione od una realizzazione, bensì è costituita dal "destinarsi" della verità dell'essere al "pensiero", e di lì al "linguaggio", in cui tale verità prenderà "dimora", per poter formare ad un tempo sia la "casa dell'essere" che dell'essere umano. Questi potrà dunque intravedere o- potremmo dire- incontrare quella esattamente nell' “apertura" in cui la verità dell'essere gli si prospetterà qui ed ora- nell' apertura che l'essere propriamente "è", quale "radura" (Lichtung), in cui esso stesso appunto si fa "presente" per l'Esserci e risulta tangibile per quest'ultimo (HEIDEGGER, 2013). Ora, se l'essenza dell'essere-uomo così svelantesi - benchè pur sempre secondo una luminositá "chiaroscurale" - nella radura dell'essere è la stessa verità dell'essere, allora Heidegger si trova qui in condizione di ribattere indirettamente alla celebre tesi di Sartre secondo cui

\begin{abstract}
prècisèment nous sommes sur un plan où il y a seleument des hommes ( L'Existencialisme est un humanisme, Paris, 1946, p.36). Se invece si pensa come in Sein und Zeit, si dovrebbe dire: prècisèment nous sommes sur un plan où il y a principalement l'Etre. Ma da dove proviene e che cos'è le plan? L' Être et le plan sono lo stesso. In Sein und Zeit è per prudenza che si dice: il y a l'Être: "si dà" l'essere. L'espressione il y a non traduce esattamente il "si dà" (es gibt) perchè "ciò" $(e s)$ che qui "dà" (gibt) è l'essere stesso. Il "dà" (gibt) indica l'essenza dell'essere che dà, concedendo la sua verità. Il darsi all'aperto, unitamente all'aperto medesimo, è l'essere stesso. [...] Questo "si dà" domina come il destino dell'essere, la cui storia perviene al linguaggio della parola dei pensatori essenziali. Per questo il pensiero che pensa entrando nella verità dell'essere è, in quanto pensiero, storico.Non c'è un pensiero "sistematico" e, accanto ad esso, per illustrarlo, una storia delle opinioni del passato. A differenza di quanto pensa Hegel, non c'è neppure solo una sistematica che possa fare della legge del proprio pensiero la legge della storia, e risolvere contemporaneamente quest'ultima nel sistema. Se pensiamo in modo più iniziale, c'è la storia dell'essere a cui appartiene il pensiero come memoria (Denken als Andenken) di questa storia, da essa fatto avvenire. (HEIDEGGER 2013, p. 61-62 e 63; corsivi miei).
\end{abstract}

Il pensiero stesso, dunque, in quanto riflette la (sulla) "storia dell'essere", è esso stesso originariamente "storico", ma giammai, in tale prospettiva, l'oggetto proprio del pensiero si svela quale prodotto oggettivo di un soggetto "sovrano" il quale infine, alla maniera del 
movimento dialettico della coscienza in Hegel, si scopra avervi presieduto già da sempre $\mathrm{o}$, in altri termini, giammai il destinatario del pensiero o della verità dell'essere, destinatario che per Heidegger coincide con l'Esserci, scopre l'oggetto stesso offerto al pensiero quale sua esperienza costitutiva, e dunque pur sempre quale tesi di una "soggettività". La verità od essenza dell'essere che si destina nell'apertura e con cui forma un tutt'uno piuttosto dirada o parzialmente rischiara "lo stesso piano" in cui si svolge 1""e-sistenza", ossia il costitutivo “essere fuori di sè" dell'essere dell'uomo; in tale prospettiva lo stesso porsi del soggetto che accede alla verità si coglie come un gettato, e mai come colui che getta, il quale altresí coincide propriamente con l'essere stesso (HEIDEGGER, 2013). Tale chiarificazione, in questo Heidegger della "svolta", del rapporto del soggetto con la verità, corrobora la già asserita analogia tra la storia della verità dell'essere inaugurata dal filosofo tedesco con la storia critica del pensiero o storia dei giochi della verità intrapresa da Foucault. Di più, se Heidegger definisce l'essere, in base allo stesso rapporto, come ciò che il pensiero propriamente "ha da pensare" (HEIDEGGER, 2013), allora tale parallelo si fa ancor più stringente alla luce di uno stralcio dello stesso Foucault, rinvenibile nell'Introduzione al secondo volume della Storia della sessualità, L'Uso dei piaceri,che essendo pubblicato nel 1984 costituisce un'ulteriore occasione di una retrospettiva globale della propria impresa per il filosofo francese; qui, non senza una certa sorpresa, Foucault reinterpreta sia le sue opere "archeologiche" che quelle "genealogiche" quali altrettante "variazioni sul tema" rispetto al modo di riferirsi reciproco di soggetto e verità, nel loro mutuo prodursi o costituirsi, e lo fa riprendendo pedissequamente la terminologia della Lettera:

C'è una certa ironia in questi sforzi per cambiare il proprio modo di vedere, per modificare l'orizzonte di ciò che si conosce e tentare di scostarsene un po'. Hanno effettivamente portato a pensare in modo diverso? Forse, hanno consentito tutt'al più di pensare diversamente ciò che già si pensava e di vedere ciò che si è fatto da un'angolazione diversa e in una luce più netta. Si credeva di allontanarsi e ci si ritrova perfettamente a piombo. Il viaggio ringiovanisce le cose e invecchia il rapporto con se stessi. Adesso, mi sembra di vedere meglio in che modo, un po' alla cieca e frammentariamente, avevo abbordato l'impresa di una storia della verità: analizzare non i comportamenti nè le idee, non le società nè le loro "ideologie", bensì le problematizzazioni attraverso le quali l'essere si dà come essere che può e deve essere pensato, e le pratiche a partire dalle quali queste problematizzazioni si formano. La dimensione archeologica dell'analisi permette di analizzare le forme stesse della problematizzazione; la sua dimensione genealogica, la loro formazione a partire dalle pratiche e dalle loro modificazioni. (FOUCAULT 2011, p. 17; corsivi miei). 


\section{III}

Ora, posto che ciò di cui "si prende cura" il pensiero - per adoperare la terminologia di Sein und Zeit (HEIDEGGER, 2014) - è pur sempre 1' "essere" anche nella visione di quest' "ultimo" Foucault, tuttavia lo stesso brano ora riportato ribadisce e al contempo complica tale analogia con l'ontologia di Heidegger. Se infatti per il filosofo francese che riflette sulla continuitá delle sue analisi la "storia della verità" si apparenta alla storia dell'essere, tuttavia quest'ultima si dà "attraverso le problematizzazioni" e le "pratiche a partire dalle quali queste [ultime] si formano": il termine "problematizzazione" a quest'altezza dell'opera foucaultiana equivale appunto a quello di evenemenzializzazione, ossia alla maniera generale e tuttavia singolare con cui il soggetto o la soggettività si esperisce ed insieme esperisce un determinato rapporto con gli oggetti e con gli altri; ma perciostesso la storia della verità in Foucault si articolerà con una "storia della soggettività" - come abbiamo visto sopra nello stralcio tratto dal Dictionnaire des philosophes -, nel mentre in Heidegger - come si evince dalla sua critica a Hegel - la storia dell'essere è intesa in diretta contrapposizione con qualsivoglia posizione originaria della verità da parte di una soggettività, o con una posizione della verità in una soggettività. Certamente, lasciando intatto l'impianto complessivo di Sein und Zeit anche a seguito della "svolta", Heidegger non toglie la possibilità per noi di intendere il Dasein come essere-nel-mondo che si auto-interpreta analiticamente, ma tale auto-interpretazione è un momento che si colloca ontologicamente dopo una pre-comprensione della totalitá del piano ove si collocano tanto l'Esserci quanto gli enti-oggetti (HEIDEGGER, 2014). Per contro, nel progetto foucaultiano della storia critica del pensiero riveste un'importanza cruciale l'esperienza che la soggettività compie in relazione a se stessa quale "oggetto per sè ", a titolo cioè di un'esperienza costitutiva del medesimo "sè" della soggettività (FOUCAULT, 1998); possiamo qui intravedere come tale impostazione della questione del soggetto ingeneri una difficoltà - se non addirittura un'aporia - nello stesso pensiero di Foucault, se teniamo presente come un'istanza tipica della produzione "archeologica" degli anni Sessanta, dall' Introduzione all'Antropologia pragmatica di Kant all'Archeologia del sapere, fosse stata esattamente quella di rinvenire delle strutture affatto empiriche e non-trascendentali dell'esperienza, e che dunque neutralizzassero un eventuale ruolo fondativo della coscienza in tale campo d'esperienza - ma se e come Foucault abbia saputo fare fronte a tale difficoltà intrinseca è senz'altro un punto controverso per l'interpretazione della stessa concezione 
foucaultiana della storia ${ }^{5}$. Fatto questo doveroso inciso, possiamo constatare la ragione teoretica di questo scarto che si presenta tra le due storie della verità in questione: essa sta esattamente nel ribaltamento operato da Foucault rispetto all'impostazione heideggeriana della questione della tecnica. Giungiamo così finalmente alla considerazione del secondo lemma del confronto prima menzionato, che dalla lettura della Lettera ci conduce alla considerazione dell'altro scritto heideggeriano fondamentale per la stessa formazione di Foucault, La questione della tecnica, appunto. Ora, sia nel primo che nel secondo scritto Heidegger definisce la tèchne come un "modo fondamentale" dell' $\alpha \lambda \eta \dot{\theta} \theta \varepsilon \iota \alpha$, ossia della verità intesa appunto nell'accezione già incontrata poco sopra di "dis-velamento" o "dis-velatezza", del concedersi della verità dell'essere all'essere dell'ente-uomo che gli si faccia incontro nella "radura" in cui consiste l'apertura stessa dell'essere; ma in tale disvelamento o concedersi della verità essenziale dell'essere la tecnica si configura pur sempre come un modo che può rimandare ad un' "essenza superiore" del "disvelamento stesso", con il quale è parimenti posto l'essere della tecnica quale "fondo" (Gestell) indefinitamente impiegabile (HEIDEGGER, 1998, p. 26). In tale essenziale essere "pro-vocato" a corrispondere ad una utilizzabilità, questo o quell'ente viene a ritrovare il proprio essere come già compreso nel "fondo" dispiegato dall' "avvento" di questa peculiare modalitá di concedersi dell'essere (HEIDEGGER, 1998, p. 12-13); e poichè si tratta del dispiegamento di una dimensione essenziale, lo stesso uomo, nella misura in cui prende parte tanto al fondo stesso degli impiegabili quanto alla suddetta pro-vocazione degli enti affinchè siano suscitati e disposti in quanto impiegabili, risulta arrischiato a degradare definitivamente in quella situazione esistenziale - nel senso già delucidato di e-sistenza- nella e per la quale si occulta definitivamente il collegamento "più originario" con l'essere per risolversi nel primato dell' ente, venendogli così meno la stessa possibilità della libertà (HEIDEGGER, 1998, p. 19 e 23), la quale a quest'altezza dell'elaborazione heideggeriana è re-interpretata, rispetto a Sein und Zeit, nei termini di una capacità di corrispondenza, da parte della meditazione umana, all' avvento con cui di volta in volta si dà l'essere (HEIDEGGER, 2013). In tal senso la stessa tecnica, quale modo essenziale del disvelamento della verità dell'essere, si configura ad un tempo come pericolo ma anche come potenziale via d'accesso ad una dimensione del disvelamento più originaria:

\footnotetext{
${ }^{5}$ Che Foucault non sia stato capace, in ultima analisi, di venire a capo di tale difficoltà strutturale del suo pensiero, è la tesi di Bèatrice Han (HAN 1998).
} 
Ciò che costituisce l'essere della tecnica minaccia il disvelamento, fa sovrastare la possibilità che ogni disvelamento si risolva nell' impiegare e che tutto si presenti solo nella disvelatezza del "fondo". L'attivitá dell'uomo non puo mai immediatamente ovviare a questo pericolo. Nessun atto dell'uomo può mai, da solo, scongiurare il pericolo. Tuttavia, la meditazione dell'uomo può considerare che tutto ciò che salva non può che avere un'essenza superiore, ma anche affine, a ciò che è messo in pericolo. Può darsi dunque che un disvelamento concesso più originariamente (ein anfanglicher gewahrtes Entbergen) sia in grado di far apparire per la prima volta ciò che salva nel mezzo dei pericolo che non tanto si manifesta, quanto piuttosto ancora si nasconde, nell'etá della tecnica? (HEIDEGGER, 1998, p. 26).

Ebbene, Foucault modifica questa prospettiva heideggeriana con la quale sono intesi i vicendevoli rapporti tra la tecnica e le posizioni della soggettività come pure tra quella e gli oggetti; e lo fa spostando il focus dell'analisi da una manifestazione generica della tecnica alle "tecniche" al plurale, quale sinonimo o complemento di quelle "pratiche" per mezzo delle quali il soggetto o la soggettività si costituisce - o, quasi rovesciando la prospettiva heideggeriana, pensando la stessa storicitá dell'essere dei soggetti quale prodotto od applicazione di quelle tecniche - ciò senza peraltro contraddire quanto egli afferma nell'Introduzione all'Uso dei piaceri, giacchè anche lì l'essere, sebbene pensato in un'inedita luce unitaria, si manifesta pur sempre "per mezzo" delle "pratiche" del sè operate dal soggetto su se stesso. In una conferenza tenuta nel 1981 al Dartmouth College, e che sia per la collocazione cronologica che per le tematica prescelta anticipa molto da vicino il corso al Collège de France del 1982 sull'Ermeneutica del soggetto, Foucault esprime questo ribaltamento della questione heideggeriana della tecnica in maniera cristallina, attestando peraltro una volta di più la filiazione diretta della sua estetica dell'esistenza all'ontologia del filosofo tedesco:

In breve, lo scopo del mio progetto è di costruire una genealogia del soggetto; il metodo è un'archeologia del sapere, e il dominio preciso dell'analisi è ciò che definirei "tecnologie" - vale a dire, l'articolazione di certe tecniche e di certi tipi di discorso sul soggetto.Vorrei aggiungere un'ultima cosa sul significato pratico di questa forma di analisi. Per Heidegger, è stato a causa di una crescente ossessione per la techne, come solo mezzo per arrivare alla comprensione degli oggetti, che l'Occidente ha perso contatto con l'Essere. Rovesciamo la questione e chiediamoci: quali tecniche e pratiche costituiscono la concezione occidentale del soggetto, dandogli la sua caratteristica separazione tra verità ed errore, libertà e costrizione? Io penso che sia qui che troveremo la possibilità reale di costruire una storia di ciò che abbiamo fatto e, al tempo stesso, una diagnosi di ciò che siamo. Si tratterebbe di un'analisi teorica dotata, al tempo stesso, di una dimensione politica. Con questa espressione, "dimensione politica", intendo un'analisi che si riferisce a ciò che siamo disposti ad accettare nel nostro mondo; ad accettare, rifiutare e cambiare sia in noi stessi, sia nella nostra situazione. In sintesi, si tratta di cercare un altro tipo di filosofia critica: non una filosofia critica che cerchi di determinare le condizioni e i limiti della nostra possibile conoscenza dell'oggetto, ma una filosofia critica che 
ricerchi le condizioni e le indefinite possibilità per trasformare il soggetto, per trasformare noi stessi. (FOUCAULT, 2012, p. 37-38; corsivi miei).

\section{IV}

La citazione per esteso è particolarmente rivelatoria, in quanto ci permette di rilevare, in Foucault, un intreccio diretto tra il rovesciamento dell'ontologia heideggeriana, da un lato, ed il rovesciamento del Criticismo kantiano dall'altro: la questione della finitudine nel senso della delimitazione universale-necessaria di ciò che possiamo "sapere", "fare" e "sperare"6 ritrova un corrispettivo, nell'analisi foucaultiana - già a partire dalla Introduzione all'Antropologia pragmatica di Kant del 1961 (FOUCAULT: In KANT, 2010) -, nel riferimento al "più iniziale" o "veramente originario" dell'essere, smarrito il quale si degraderebbe in un dominio conoscitivo inautentico ed alienato. Al contrario, per il filosofo francese si tratta di muovere dalla positività delle pratiche o tecniche del sè secondo la volontà decisoria del soggetto di trasformarsi secondo ciò che egli giudichi preferibile per sè, per il proprio essere-nel-mondo, secondo quel criterio estetico che egli esplicita in Che cos'è l'Illuminismo (FOUCAULT: In FOUCAULT, 1998) ${ }^{7}$. In tal senso riusciamo a comprendere anche una possibile peculiare ripresa, da parte del filosofo francese, del programma heideggeriano di una ridefinizione del legame tra ontologia ed etica, programma in parte annunciato dal filosofo tedesco a Beaufret nel seguente stralcio:

Bisogna porre l'humanitas al servizio della verità dell'essere, ma senza l'umanismo
nel senso metafisico. Ma se l'humanitas è così essenziale al pensiero dell'essere,
non è allora necessario completare l' "ontologia" con un' "etica"? Non è allora
assolutamente essenziale lo sforzo che Lei esprime nella frase: "Ce que je cherche à
faire, depuis long temps dèjà, c'est prèciser le rapport de l'ontologie avec une
èthique possible?" (HEIDEGGER, 2013, p. 88).

Come per lo Heidegger della Lettera, anche per Foucault si tratta di ripensare un'etica all'altezza della realtà dispiegata della tecnica, ma non più nel senso di un "raccoglimento" in una più alta ed originaria dimensione del vero, bensì, all'opposto, nel senso di cogliere e praticare- o meglio, di cogliere praticando- degli stili di esistenza, delle tecniche del sè nelle

\footnotetext{
${ }^{6}$ Cfr. A questo proposito la celebre lettera di Kant a Carl Friedrich Staudlin, contenuta in KANT, Immanuel. Epistolario filosofico 1761-1800. Traduzione di O. Meo. Genova: Il melangolo, 1990, p. 319-320. Tale lettera veniva allegata dal l'autore alla prima edizione de La religione entro i limiti della sola ragione.

${ }^{7}$ Vedi in proposito supra, nota 1.

8 "Ma ció che cerco di fare, giá da lungo tempo, è di precisare il rapporto tra un' ontologia ed un'etica possibile"
} 
quali e con le quali il soggetto ad un tempo pone se stesso e si impartisce un insieme di prescrizioni per la prova dei propri limiti- e del loro possibile superamento.

\section{RIFERIMENTI}

ERIBON, Didier. Michel Foucault 1926-1984. Traduzione in portoghese di J.L.Gomes. Lisboa: Edição “Livros do Brasil”, 1990.

FOUCAULT, Michel. Che cos'è l'Illuminismo? (1983). In: FOUCAULT, Michel. Archivio Foucault 3. Traduzioni di Sabrina Loriga. Milano: Feltrinelli, 1997, p. 253-261.

FOUCAULT, Michel. Che cos'è l'Illuminismo? (1984). In: FOUCAULT, Michel. Archivio Foucault 3. Traduzioni di Sabrina Loriga. Milano: Feltrinelli, 1997, p. 217-232.

FOUCAULT, Michel. Foucault (1984). In: FOUCAULT, Michel. Archivio Foucault 3. Traduzioni di Sabrina Loriga. Milano: Feltrinelli, 1997, p. 248-252.

FOUCAULT, Michel. Illuminismo e Critica. Traduzione di Paolo Napoli. Roma: Donzelli Editore, 1997.

FOUCAULT, Michel. Il ritorno della morale (1984). In: FOUCAULT, Michel. Archivio Foucault 3. Traduzioni di Sabrina Loriga. Milano: Feltrinelli, 1997, p. 262-272.

FOUCAULT, Michel. Inroduzione. Traduzione di Mauro Bertani. In: KANT, Immanuel. Antropologia da un punto di vista pragmatico.Traduzione di Gianluca Garelli. Torino: Einaudi, 2010. Introduzione, p. 9-94.

FOUCAULT, Michel. L'Ermeneutica del soggetto. Corso al Collège de France 1981-1982. Traduzione di Mauro Bertani. Milano: Feltrinelli, 2004.

FOUCAULT, Michel. L'Uso dei Piaceri. Traduzione di Laura Guarino. Milano: Feltrinelli, 2011.

FOUCAULT, Michel. Sull'origine dell'ermeneutica del sè.Traduzioni a cura di Materiali Foucaultiani. Napoli: Cronopio, 2012.

HAN, Bèatrice. L’ontologie manquee de Michel Foucault. Grenoble: Millon, 1998.

HEIDEGGER, Martin. Essere e tempo (1927). Traduzione di Franco Volpi. Milano: Longanesi, 2014.

HEIDEGGER, Martin. La questione della tecnica (1953). In HEIDEGGER, Martin. Saggi e discorsi. Traduzioni di Gianni Vattimo. Milano: Mursia, 1998.

HEIDEGGER, Martin. Lettera sull' "umanismo" (1946). Traduzione di Franco Volpi. Milano: Adelphi, 2013. 